\title{
Sunspot Time Series Forecasting using Deep Learning
}

\author{
Mahmoud Elgamal
}

\begin{abstract}
In order to forecast solar cycle 25, sunspot numbers(SSN) from $1700 \sim 2018$ was used as a time series to predict the next eleven years. deep long short-term memory(LSTM) was exploited to do the forecast, first the dataset was split into training set(80\%) and (20\%) for the test set, the achieved accuracy led us to forecast the next eleven years. The result shows that the cycle will be from $2019 \sim 2029$ with peak at 2024.
\end{abstract}

\section{Introduction}

One interesting aspect of the Sun is its sunspots. Solar flares, coronal mass ejections, high-speed solar wind, and solar energetic particles are all forms of solar activity. All solar activity is driven by the solar magnetic field[14].

Solar activity has important influences on all living beings, and major technologies in the world. As a result of the changes in solar activity at a period of about 11 years, deviations in the near-Earth space in the interplanetary environment and the magnetosphere change the ionospheric plasma density with this period. Furthermore, changes in solar activity affect aero and space navigation,spaceflights, radars, high-frequency radio communications, and ground electrical lines. These changes also may affect climate and living organisms on the earth, including humans([1, 10, 11]). In order to estimate the sunspot cycle, sunspot number(SSN) is one of the main used indices, it is an important parameter in many scientific areas $([13,7])$. The sunspot number is an active field of research, because of its importance, difficulty of estimation, and possible applications based on it.

A time series is a set of observations, each one being recorded at a specific time. Time series prediction is a vital research area in which forecasters collect and analyze historical observations to identify a model to capture the underlying data generation process, this model will be used to predict the future values. In the last decades Artificial neural networks(ANN) proved to be a serious contenders to statistical methods in forecasting. In order to use 
ANN in forecasting tasks, it must compromise among three issues: solution complexity, forecast accuracy, and data characteristics[16].

Recurrent neural network(RNN) found to be the convenient one to achieve the above mentioned tasks, yet the majority weakness of RNN is vanishing/exploding gradient problem[3]. In [8, 12] this drawback was handled using long short-term memory(LSTM) algorithm as an extension to RNN. The paper is composed of: LSTM model section(2), Deep-LSTM model section(3), and simulation in section(4).

\section{LSTM Model}

Recurrent neural networks(RNN)found to be convenient method in forecasting time series, as it compromises among solution complexity, forecast accuracy, and data characteristics[16]. RNN have the ability to store memory since their current output is dependent on the previous computations. However, the major weakness of RNN is the vanishing/explosion gradient problem[5]. Long short-term memory(LSTM) were designed to overcome vanishing/explosion problem by introducing new gates which allow a better control over the gradient flow and enable better presentation of long range dependencies. Figure(1) shows the memory cell of LSTM with three gates: input gate, forget gate, and output gate.

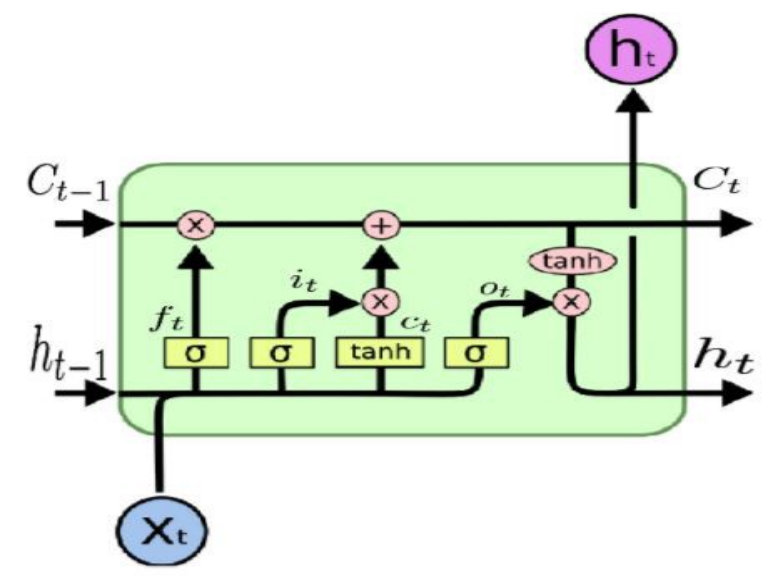

Figure 1: LSTM cell structure.

The input sequence $\left\{x_{1}, x_{2}, \cdots, x_{n}\right\}$ is input to RNN model using recurrence relation:

$$
h_{t}=f\left(h_{t-1}, x_{t}\right) \text {, }
$$


where $x_{t} \equiv$ input at time $t, h_{t} \equiv$ current hidden state.

LSTM overcomes vanishing/explosion problem by introducing the gates and states of LSTM are computed as follows:

input gate:

$$
i_{t}=\sigma\left(W_{1}^{i} \cdot x_{t}+W_{h}^{i} \cdot h_{t-1}+b_{i}\right)
$$

forget gate:

$$
f_{t}=\sigma\left(W_{1}^{f} \cdot x_{t}+W_{h}^{f} \cdot h_{t-1}+b_{f}\right),
$$

output gate:

$$
o_{t}=\sigma\left(W_{1}^{o} \cdot x_{t}+W_{h}^{o} \cdot h_{t-1}+b_{o}\right)
$$

cell input:

$$
\bar{C}_{t}=\tanh \left(W_{1}^{C} \cdot x_{t}+W_{h}^{C} \cdot h_{t-1}+b_{C}\right),
$$

where $W_{1}^{i}, W_{1}^{f}, W_{1}^{o}, W_{1}^{C}$ are the weight matrices connecting $x_{t}$ to the three gates and the cell input, $W_{h}^{i}, W_{h}^{f}, W_{h}^{o}, W_{h}^{C}$ are the weight matrices connecting $h_{t-1}$ to the three gates and the cell input, $b_{i}, b_{f}, b_{o}, b_{C}$ are the bias terms of the three gates and the cell input, $\sigma$ represents the Sigmoid function and tanh represents the hyperbolic tangent function. Secondly, calculate the cell output state:

$$
C_{t}=i_{t} * \bar{C}_{t}+f_{t} * C_{t-1},
$$

where $i_{t}, f_{t}, \bar{C}_{t}, C_{t-1}$ and $C_{t}$ have the same dimension. Thirdly, calculate the hidden layer output:

$$
h_{t}=o_{t} * \tanh \left(C_{t}\right) .
$$

To predict the future value $y_{t}$, we use the observed historical data $x_{t}$ as the network input(see figure(2)). From the above LSTM calculations, $h_{t}$ is obtained and hence the network output is

$$
y_{t}=W_{2} \cdot h_{t}+b
$$

where $W_{2}$ is the weight matrix between the output layer and the hidden layer, $b$ is the bias term of the output layer. 


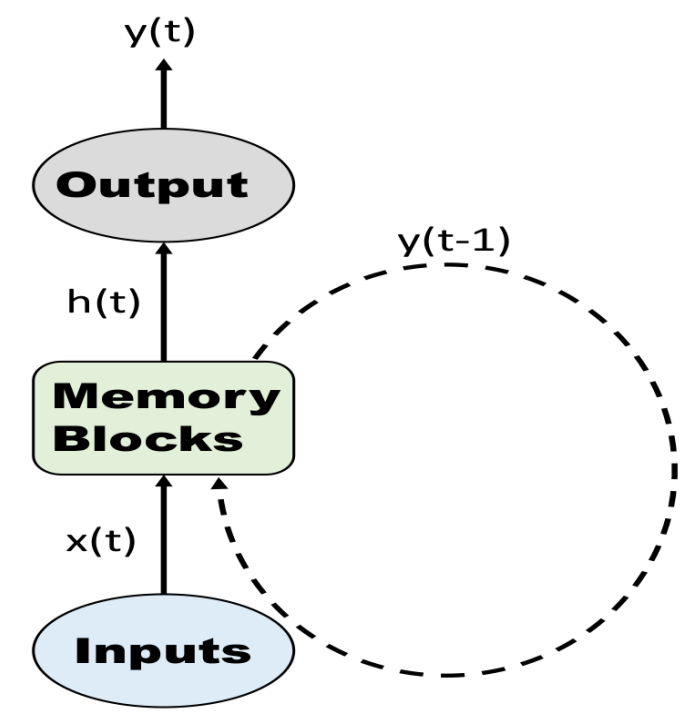

Figure 2: LSTM structure for time series prediction.

\section{Deep LSTMs}

Stacked LSTMs or Deep LSTMs were introduced by Graves[4], et al. in their application of LSTMs to speech recognition, beating a benchmark on a challenging standard problem. They found that the depth of the network was more important than the number of memory cells in a given layer to model skill. Stacked LSTMs are now a stable technique for challenging sequence prediction problems. A Stacked LSTM architecture(figure(3)) can be defined as an LSTM model comprised of multiple LSTM layers. An LSTM layer above provides a sequence output rather than a single value output to the LSTM layer below. Specifically, one output per input time step, rather than one output time step for all input time steps.

To increase efficiency of the LSTM networks, several stacked blocks of LSTM were used in a similar way to deep recurrent network[2].

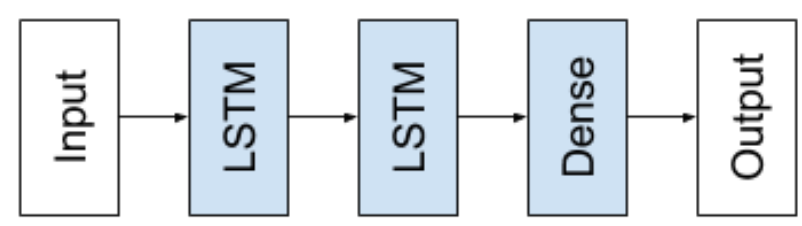

Figure 3: Stacked Long Short-Term Memory Architecture. 


\section{Experiments}

The dataset of the sunspot number are freely available from world data center SILSO[15] for daily, monthly, and yearly in csv-format. The simulation run on yearly dataset from year 1700 till 2108, i.e., 318 years.

\subsection{Forecasting Accuracy Errors}

To measure forecast accuracy and performance evaluation, the commonly used measures are[6]:

- Root Mean Squared Error(RMSE) easy to interpret your model accuracy written as:

$$
\mathrm{RMSE}=\sqrt{\frac{1}{n} \sum_{i=1}^{n}\left(y_{i}-\hat{y}_{i}\right)^{2}}
$$

- Another commonly used one is Root Mean Square Percentage Er$\operatorname{ror}(\mathrm{RMSPE})$

$$
\mathrm{RMSPE}=\sqrt{\frac{1}{n} \sum_{i=1}^{n}\left(\frac{y_{i}-\hat{y}_{i}}{y_{i}}\right)^{2}} \times 100
$$

- MAPE refers to Mean Absolute Percentage Error, which is

$$
\mathrm{MAPE}=\frac{100}{n} \sum_{i}^{n} \frac{y_{i}-\hat{y}_{i}}{y_{i}}
$$

where $y_{i}$ is the true value(observation) and $\hat{y}_{i}$ is the predicted value.

\subsection{Results}

The SSN dataset from $1700 \sim 2018$ was split into $80 \%$ for training set and ( $20 \% \approx 64$ years) for test set. Then we run the deep LSTM program with configurations in table(1) to get the result shown in figure(4)

Table 1: Deep LSTM configurations.

\begin{tabular}{|c|c|c|c|c|c|}
\hline No. of layer & No. of hidden units in each layer & No. of Epochs & RMSE & RMSPE & MAPE \\
\hline 2 & {$[5,5]$} & 1000 & 23.478 & 58.383 & 32.042 \\
\hline \hline
\end{tabular}




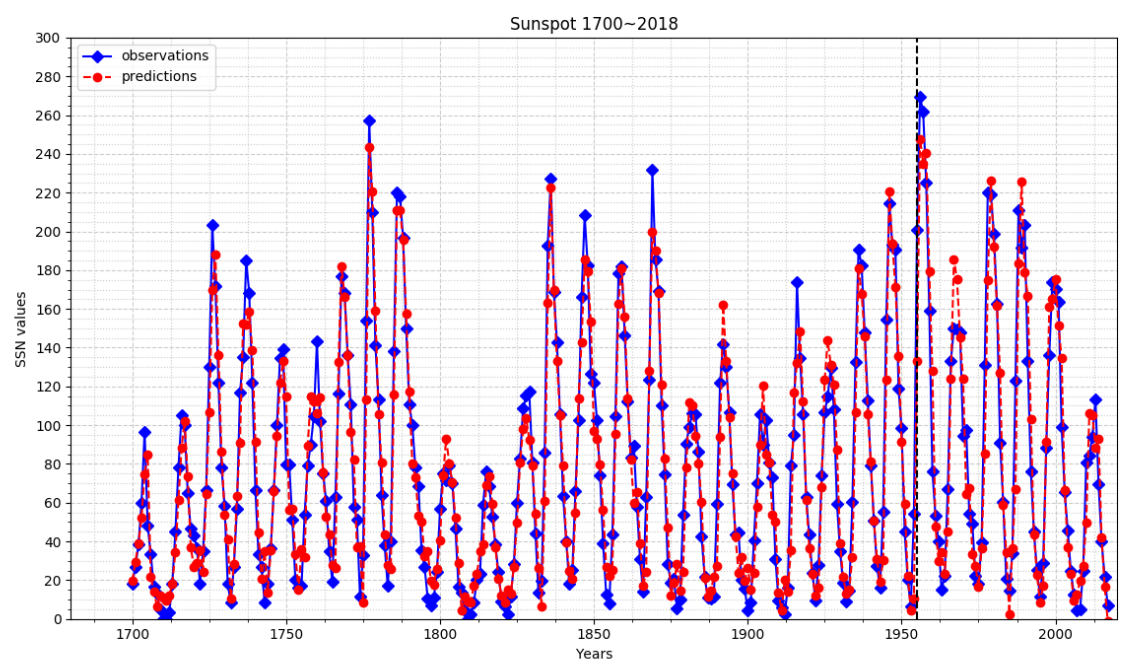

Figure 4: SSN and their forecasts.

It is important to predict the next solar cycle, so the deep LSTM model run to predict the future eleven years and the results shown in figure(5) and table(2). It is clear that solar cycle 25 will have a peak SSN of 120 at year 2024.

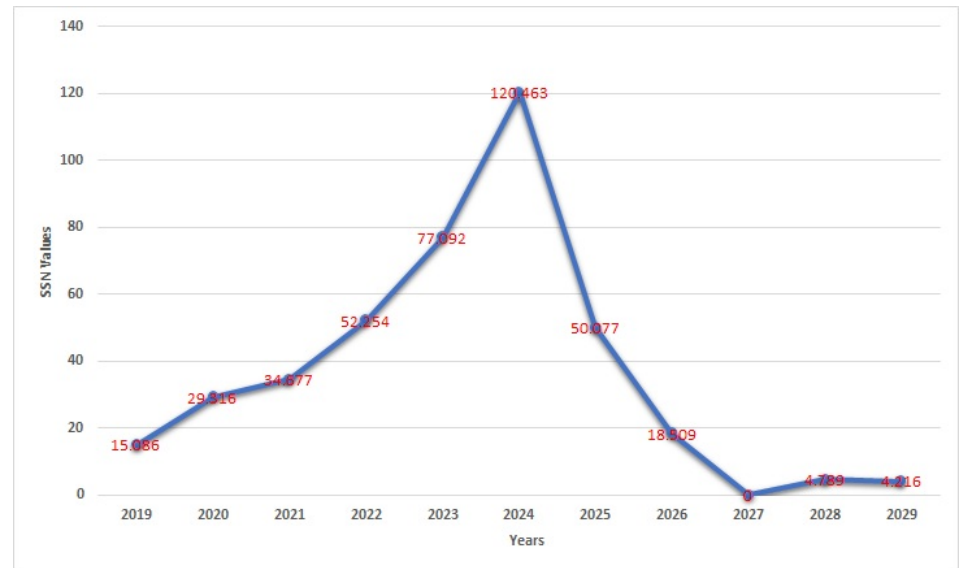

Figure 5: SSN and their forecasts for the next 11 years. 
Table 2: SSN numbers and their predicted values.

\begin{tabular}{||c||c||}
\hline \hline Year & SSN value \\
\hline 2019 & 15.086 \\
\hline 2020 & 29.316 \\
\hline 2021 & 34.677 \\
\hline 2022 & 52.254 \\
\hline 2023 & 77.092 \\
\hline 2024 & 120.463 \\
\hline 2025 & 50.077 \\
\hline 2026 & 18.509 \\
\hline 2027 & 0 \\
\hline 2028 & 4.789 \\
\hline 2029 & 4.216 \\
\hline \hline
\end{tabular}

\section{Conclusion}

In this paper, solar cycle 25 was studied using deep LSTM, first data split into training/test sets with $80 \% / 20 \%$ and after choosing the right parameters it was run to predict the next solar cycle.

\section{References}

[1] L. A. Aguirre, C. Letellier, and J. Maquet, Forecasting the Time Series of Sunspot Numbers, Solar Physics volume 249, pages103-120(2008).

[2] j. Brownlee, Stacked Long Short-Term Memory Networks, 2019.

[3] Bayer, J. Simon, learning sequence representations, Technischen Universität München, 2015.

[4] A. Graves, A. R. Mohamed, and G. Hinton, Speech recognition with deep recurrent neural networks. In Proc. International Conference on Acoustics, Speech and Signal Processing 6645-6649 (2013).

[5] S. Hochreiter, "The Vanishing Gradient Problem During Learning Recurrent Neural Nets and Problem Solutions", International Journal of Uncertainty, Fuzziness and Knowledge-Based SystemsVol. 06, No. 02, pp. 107-116 (1998). 
[6] R. J. Hyndman, and A. B. Koehler Another look at measures of forecast accuracy,International Journal of Forecasting 22 (2006) $679-688$.

[7] K.B. Kim, J.H. Kim, H.Y. Chang, Do Solar Cycles Share Spectral Properties with Tropical Cyclones that Occur in the Western North Pacific Ocean?, J. Astron. Space Sci. 35, 151, 2018.

[8] Pascanu, Razvan, T. Mikolov, On the difficulty of training recurrent neural networks, Proceedings of the 30th International Conference on Machine Learning(3), volume 28, 2013, pp. 1310-1318.

[9] J. Patterson, A. Gibson, Deep Learning. A Practitioner's Approach; O'Reilly Media, Inc.: Sebastopol, CA, USA, 2017; pp. 150-158.

[10] W. D. Pesnell, Predictions of Solar Cycle 24, Solar Physics volume 252, pages209-220(2008).

[11] K. Petrovay, Solar Cycle Prediction, Living Reviews in Solar Physics volume 7, Article number: 6 (2010).

[12] J. Sutskever, Training recurrent neural networks, university of Toronto,PhD thesis, 2012.

[13] S. Sagir, S. Karatay, R. Atici, A. Yesil, O. Ozcan, The relationship between the Quasi Biennial Oscillation and Sunspot Number, Adv. Space Res. 55, 106.,2015.

[14] What is solar activity?, National Aeronautics and Space Administration, 2017.

[15] World Data Center for the production, preservation and dissemination of the international sunspot number, http://www.sidc.be/silso/datafiles, 2019 .

[16] G. Zhang, B.E. Patuwo, M. Y. Hu, Forecasting with artificial neural networks:: The state of the art, International Journal of Forecasting Volume 14, Issue 1, 1 March 1998, Pages 35-62. 